Published in final edited form as:

J Am Chem Soc. 2016 March 30; 138(12): 4219-4228. doi:10.1021/jacs.6b00633.

\title{
Molecular Characterization of the Cercosporin Biosynthetic Pathway in the Fungal Plant Pathogen Cercospora nicotianae
}

\author{
Adam G. Newman and Craig A. Townsend ${ }^{*}$ \\ Department of Chemistry, Johns Hopkins University, Baltimore, MD, 21218, USA
}

\begin{abstract}
Perylenequinones are a class of photoactivated polyketide mycotoxins produced by fungal plant pathogens that notably produce reactive oxygen species with visible light. The best-studied perylenequinone is cercosporin - a product of the Cercospora species. While the cercosporin biosynthetic gene cluster has been described in the tobacco pathogen Cercospora nicotianae, little is known of the metabolite's biosynthesis. Furthermore, in vitro investigations of the polyketide synthase central to cercosporin biosynthesis identified the naphthopyrone nor-toralactone as its direct product - an observation in conflict with published biosynthetic proposals. Here, we present an alternative biosynthetic pathway to cercosporin based on metabolites characterized from a series of biosynthetic gene knockouts. We show that nor-toralactone is the key polyketide intermediate and the substrate for the unusual didomain protein CTB3. We demonstrate the unique oxidative cleavage activity of the CTB3 monooxygenase domain in vitro. These data advance our understanding of perylenequinone biosynthesis and expand the biochemical repertoire of flavindependent monooxygenases.
\end{abstract}

\section{INTRODUCTION}

The plant pathogenic Cercospora species are a widespread and destructive genus of ascomycetous fungi characterized by their production of the phytotoxin cercosporin (1, Figure 1b). ${ }^{1}$ Cercosporin belongs to the perylenequinone natural product family. The perylenequinone metabolites share a characteristic core architecture (2) that is essential to their toxicity. ${ }^{2,3}$ Multiple perylenequinone natural products have been identified from fungal and aphidian sources. ${ }^{4}$ Cercosporin was first isolated in 1957 from Cercospora kikuchii T. Matsu \& Tomoyasu—a fungal pathogen of soybeans. ${ }^{5,6}$ Perylenequinone metabolites contain the same highly oxidized conjugated pentacyclic core regardless of varied substituents-primarily at positions $\mathrm{C} 7$ and $\mathrm{C}^{\prime}{ }^{\prime}$ —and are typically $C_{2}$-symmetric. Notably, most perylenequinone metabolites are helically chiral, demonstrating a preference for one particular atropisomer despite their conjugated, $\mathrm{sp}^{2}$-hybridized carbon core. ${ }^{7-9}$ Steric clashes between substituents in the mature dimeric metabolites account for the observed helical chirality. The biosynthetic origin of this property is unknown.

Corresponding Author: ctownsend@jhu.edu.

Supporting Information

Experimental details, CTB3 and CTB2 sequence details, full results of complementation assay and CTB3 + CTB2 in vitro reactions, and compound spectra. This material is available free of charge on the Internet via http://pubs.acs.org. 
Cercosporin-like all perylenequinone metabolites-functions as a photosensitizing agent. ${ }^{10}$ Upon absorption of visible light, cercosporin facilitates remarkably efficient transfer of this energy to $\mathrm{O}_{2}$ (quantum yield $0.81^{11}$ ), leading to the production of the potent reactive oxygen species (ROS) singlet oxygen $\left({ }^{1} \mathrm{O}_{2}\right)$ and superoxide radical $\left(\mathrm{O}_{2}{ }^{\bullet-}\right)$. These ROS cause indiscriminate damage to a variety of cellular targets including cell membranes, nucleic acids, proteins, and lipids. ${ }^{12-14}$ Peroxidation of the cell membrane is particularly pernicious and is the primary mode of toxicity, causing ion leakage from the host organism. ${ }^{12,13} \mathrm{~A}$ direct cellular target of cercosporin has not been found but it is believed that its toxicity is entirely attributed to indiscriminant damage by ROS. As a result, cercosporin exerts broad toxicity to bacteria, fungi, and mice. ${ }^{15-17}$ Production of cercosporin by Cercospora nicotianae - a fungal pathogen of tobacco — is crucial to the fungus's pathogenicity and is concomitant with lesion formation on tobacco leaves. ${ }^{18-20}$ Multiple putative resistance mechanisms have been proposed in $C$. nicotianae, but the primary mode of resistance is through reversible reduction of the perylenequinone moiety to dihydrocercosporin (3) - a species with little photosensitizing capacity. Dihydrocercopsorin is oxidized to cercosporin spontaneously upon export from the fungus, restoring its photoactivated toxicity. ${ }^{21-23}$

Cercosporin was classified early on as a polyketide natural product and bore the characteristic alternating polyketide labeling pattern from acetyl and malonyl building blocks. ${ }^{24}$ Eventually a gene from $C$. nicotianae encoding a fungal polyketide synthasedubbed CTB1 (cercosporin toxin biosynthesis 1) —was identified through restriction enzyme-mediated integration mutagenesis. ${ }^{18}$ CTB 1 is absolutely necessary for cercosporin production and bears all the hallmarks of an iterative, non-reducing polyketide synthase (NR-PKS). ${ }^{25}$ Using CTB1 as a benchmark, the complete cercosporin biosynthetic gene cluster from $C$. nicotianae was determined (Figure 1a, Table 1). ${ }^{26}$ The cluster comprises eight genes, six of which are believed to be responsible for cercosporin assembly (CTB1, 2, $3,5,6$, and 7). ${ }^{18,20,26,27}$ The zinc finger transcription factor CTB8 co-regulates expression of the cluster, ${ }^{26}$ while the major facilitator superfamily (MFS) transporter CTB4 exports the final metabolite. ${ }^{19}$ In addition to the identified gene cluster, the $\mathrm{Zn}(\mathrm{II}) \mathrm{Cys}_{6}$ transcription factor CRG1 is implicated in both cercosporin production and resistance. ${ }^{28-30}$ The $\mathrm{Zn}(\mathrm{II}) \mathrm{Cys}_{6}$ family of transcription factors is unique to fungi that regulate diverse cellular processes. ${ }^{31}$ Regulation by CRG1 is complex and poorly understood; however, its expression is implicated in regulation of chemical detoxification, multidrug membrane transport, and antioxidant biosynthesis pathways. ${ }^{30}$ Cercosporin production is completely dependent upon exposure to light, and $C$. kikuchii grown in the dark will not accumulate cercosporin. ${ }^{32}$ The regulatory mechanism governing light dependence is unknown.

On the basis of individual homologies of the $C$. nicotianae gene cluster and retrobiosynthetic analysis, Chen and coworkers proposed a biosynthetic pathway for cercosporin (Figure 1b). ${ }^{26}$ This proposal hinges upon the metabolite's $C_{2}$-symmetry and the authors argued that dimerization of two identical aromatic intermediates $\mathbf{4}$ would lead to the perylenequinone core. They postulated that CTB1 produces carboxylic acid $\mathbf{5}$ from which the aromatic intermediate 4 is derived. While this biogenesis appears reasonable and accounts for the putative activity of all the biosynthetic gene cluster members, it is nevertheless likely incorrect. Using an enzyme-deconstruction approach, we previously characterized the in 
vitro activity of CTB1. ${ }^{25}$ Unexpectedly, the naphthopyrone nor-toralactone (6) is the unambiguous in vitro product of CTB1 (Figure 1b). The identification of this intermediate is problematic, as there is no clear member of the gene cluster that could presumably open the pyrone moiety - an event that must occur to access the perylenequinone core architecture. We present an alternative biosynthetic pathway bolstered by metabolites accumulated in pathway-interrupted $C$. nicotianae knockouts. Furthermore, we characterize the in vitro activity of CTB3 - an unusual didomain protein containing $O$-methyltransferase and flavindependent monooxygenase domains — and demonstrate its role in toralactone formation — an $O$-methylated congener of nor-toralactone-and subsequent pyrone opening.

\section{RESULTS}

\section{Chemical Identification of Intermediates}

C. nicotianae has been previously cultivated in liquid potato dextrose broth (PDB) and on solid PDA. While functional cercosporin biosynthetic gene knockout strains of $C$. nicotianae grew in PDB, reproducible metabolite profiles could not be obtained. Therefore, metabolites were isolated from cultures grown on PDA under constant light, which provided reproducible metabolite profiles. Cultures of wild-type $C$. nicotianae resulted in a clean metabolic profile with the red-pigmented cercosporin (1) as the principal product (Figure 2a). Cercosporin was absent in the HPLC profiles of all functional cercosporin biosynthetic gene knockout strains. As expected, secondary metabolite accumulation was missing in the $\triangle \mathrm{CTB} 1$ mutant - the knockout mutant of the central NR-PKS of cercosporin biosynthesis (Figure 2b).

The structures of the major accumulated metabolites from $C$. nicotianae CTB gene cluster knockout strains were elucidated by characteristic UV spectra, exact masses, mass fragmentation patterns, and where possible NMR spectra. We identified the previously characterized naphthopyrones nor-toralactone (6) and toralactone (7) both as metabolites of the $\triangle \mathrm{CTB} 3 \mathrm{c}$ mutant (Figure $2 \mathrm{~d}$ ). Also accumulated in the $\Delta \mathrm{CTB} 3 \mathrm{c}$ mutant was the oxidation product of nor-toralactone, naphthoquinone 8. Two previously unobserved naphthoquinones were isolated from the $\triangle \mathrm{CTB} 6$ and $\triangle \mathrm{CTB} 5$ mutants, which we called cercoquinone $\mathrm{A}(\mathbf{9})$ and cercoquinone B (10), respectively (Figure $2 \mathrm{f}, \mathrm{e}$ ). No major compounds were observed in the extracted metabolite profiles for the $\triangle \mathrm{CTB} 2$ and $\triangle \mathrm{CTB} 7$ mutants (Figure $2 \mathrm{c}, \mathrm{g}$ ). The profiles for each of these mutants were similar to that of the $\triangle \mathrm{CTB} 1$ mutant. Proposed structural assignments for new compounds cercoquinones $\mathrm{A}$ and $\mathrm{B}$ are supported by their ${ }^{1} \mathrm{H}$ NMR spectra, HRMS, mass fragmentation patterns and UV spectra (see Supporting Information).

\section{Phenotypic Analysis of Gene Knockout Strains}

The mycelia of wild-type $C$. nicotianae were blood red in color, with pigmentation occurring at about 4 days after inoculation (Figure 2a). The pigmented metabolites were primarily concentrated in the mycelia with a small amount exported into the agar surrounding individual colonies. The mycelia of each knockout strain displayed a different pigmentation from wild-type $C$. nicotianae, with pigmentation similarly occurring at about 4 days after inoculation. The $\triangle \mathrm{CTB} 1$ mutant did not display any pigmentation (Figure 2b). The $\Delta \mathrm{CTB} 3 \mathrm{c}$ 
mutant adopted a dark yellow-brown coloration, with slight export of pigmented metabolites into the agar (Figure 2d). The mycelia of both $\triangle \mathrm{CTB} 6$ and $\triangle \mathrm{CTB} 5$ mutants turned a dark orange-red with significant export of colored compounds into the agar (Figure 2f,e). Although they did not accumulate any observed extractable secondary metabolite observed by HPLC, the mycelia of the $\triangle \mathrm{CTB} 2$ and $\triangle \mathrm{CTB} 7$ mutants both adopted a yellow-brown color with some export of pigmented compounds into the agar (Figure 2c,g).

In addition to the pigmentation phenotypes, the average colony diameter for each individual strain was distinctive after 7 days of growth (Figure 3b). The average colony diameters for the wild-type, $\triangle \mathrm{CTB} 3 \mathrm{c}$, and $\Delta \mathrm{CTB} 6$ strains were the smallest at $15.5 \pm 0.8,15.7 \pm 1.1$, and $15.9 \pm 1.0 \mathrm{~mm}$ (average \pm standard deviation, $\mathrm{n}=37$ ). The average colony diameter for the $\Delta$ CTB5 mutant was of intermediate size at $17.1 \pm 1.1 \mathrm{~mm}$, while the average colony diameters for the $\triangle \mathrm{CTB} 1, \triangle \mathrm{CTB} 2$, and $\triangle \mathrm{CTB} 7$ mutants were the largest at $20.8 \pm 1.3,19.2$ \pm 1.4 , and $18.7 \pm 1.8 \mathrm{~mm}$, respectively. The average colony diameter showed an inverse correlation with small molecule accumulation; the strains with identifiable cercosporin metabolites (wild-type, $\triangle \mathrm{CTB} 3 \mathrm{c}, \Delta \mathrm{CTB} 6$, and $\Delta \mathrm{CTB} 5$ ) displayed smaller colony diameters while the strains without identifiable extractable intermediates $(\triangle \mathrm{CTB} 1, \Delta \mathrm{CTB} 2$, and $\Delta$ CTB7) displayed larger colony diameters.

\section{Cercosporin Complementation Assay}

To test whether accumulated metabolites represented on-pathway intermediates, we conducted a complementation assay in which pairs of CTB gene cluster knockout strains were grown adjacent to one another. Cercosporin biosynthetic complementation was indicated by red pigmentation at the colony-colony interface. The only pair that could successfully complement cercosporin biosynthesis was the $\triangle \mathrm{CTB} 1$ and $\triangle \mathrm{CTB} 3 \mathrm{c}$ mutant pair (Figure 3a). Clear complementation was observed between 4 and 5 days after inoculation between colonies spotted approximately $0.5 \mathrm{~cm}$ apart. Pigmentation was isolated to the $\triangle \mathrm{CTB} 1$ mutant with no clear accumulation of cercosporin in the $\triangle \mathrm{CTB} 3 \mathrm{c}$ mutant. We attempted to detect cercosporin extracted from agar plugs of the colony-colony interface using a previously described spectrophotometric assay ${ }^{33}$; however, the amount of accumulated cercosporin was below the detection limit. No other mutant pair showed clear cercosporin complementation (Figure S3, Supporting Information).

\section{Genetic Analysis of the Cercosporin Gene Cluster}

The antiSMASH algorithm was used to identify gene clusters homologous to the CTB gene cluster. ${ }^{34}$ The top five sequences all came from fungal plant pathogens (Figure $3 \mathrm{c}$ ). Of these sequences, only one was associated with a known natural product, the hypocrellin A biosynthetic cluster from Shiraia sp. slf14 responsible for the formation of the perylenequinone hypocrellin A (13). ${ }^{35}$ Five homologous CTB genes were shared among all identified clusters: homologs of CTB1, 3, 2, 5, and 4. The CTB1 homologs contained the canonical NR-PKS domain architecture as determined by a BLASTp search. All of the homologs of CTB3 displayed the same unique didomain architecture with a predicted $\mathrm{N}$ terminal $O$-methyltransferase and a $C$-terminal flavin-dependent monooxygenase. 


\section{In Vitro Analysis of CTB3 Activity}

Initially deducing that it followed $\mathrm{CTB} 1$ on the cercosporin pathway, we investigated the in vitro activity of CTB3 toward nor-toralactone-the in vitro product of CTB1. Because of its unique didomain architecture, we dissected CTB3 into its constituent domains- the $N$ terminal $O$-methyltransferase (CTB3-MT) and the $C$-terminal flavin-dependent monooxygenase (CTB3-MO) - for ease of expression and to investigate the activity of each domain individually (Figure S1, Supporting Information). Both CTB3 and CTB3-MO were purified as holo enzymes with the FAD cofactor bound to the proteins. The presence of FAD was confirmed by HPLC and spectrophotometric analysis (Figure S2, Supporting Information). Cosubstrates SAM and NADH were included in reactions of CTB3 as needed. SAM served as the methyl donor for CTB3-MT and NADH served as the reductant for CTB3-MO-transferring a hydride equivalent to FAD at the initiation of a catalytic cycle.

In vitro reactions were conducted with both nor-toralactone (6) and toralactone (7) as substrates. Initial reactions containing $10 \mu \mathrm{M}$ CTB3 clearly processed these substrates; however, the product profiles were ambiguous. Furthermore, the CTB3-MO domain processed both potential substrates with nor-toralactone being converted to a host of products. While these species were not fully characterized, MS and UV data were indicative of perylenequinone-like products with the clear loss of a single carbon. In order to simplify analysis of these enzymatic reactions, significant effort went into optimizing the experimental conditions. We found that lower protein concentrations $(1 \mu \mathrm{M})$ and simplified sample preparation (filtration only before HPLC analysis) were necessary for reproducible and robust analysis.

Reactions of CTB3-MT showed turnover of nor-toralactone to toralactone (Figure 4a). Similarly, reactions containing a low concentration of protein $(0.1 \mu \mathrm{M})$ showed partial methylation of nor-toralactone to toralactone. Together, these results indicated that toralactone served as an intermediate of the CTB 3 catalytic cycle and was the true substrate for the CTB3-MO domain despite its apparent activity towards nor-toralactone. Postulating that the programming of the catalytic cycle between the MT and MO domains was in part kinetically controlled, we conducted reactions that were allowed to proceed for $60 \mathrm{~min}$ before quenching - significantly longer than the 7-10 min reactions initially employed. Reactions of the CTB3-MO domain with toralactone serving as the substrate resulted in turnover to $o$-naphthoquinone 12-cercoquinone C (Figure 4b). Cercoquinone $\mathrm{C}$ was also observed in reactions of deconstructed CTB3 with nor-toralactone as the substrate-albeit with a coterie of other uncharacterized products (Figure 4c). These additional products were absent in reactions with toralactone. Additionally, toralactone is an observed intermediate of this reaction along with the off-pathway oxidation product, naphthoquinone 8 .

The formation of cercoquinone $\mathrm{C}$ was unexpected. Presumably, this product is formed through its hydroquinone (14) even though that species was not directly observed. Assuming that oxidation was spontaneous, we postulated that conducting in vitro reactions under reductive conditions could have prevented the presumptive oxidation. Reactions under reductive conditions (1 $\mathrm{mM}$ DTT) resulted in the formation of a new $p$-naphthoquinone 11cercoquinone D (Figure 4d-f). Cercoquinone D was only observed when DTT was present.

J Am Chem Soc. Author manuscript; available in PMC 2016 November 30. 
It was observed in either reactions of combined CTB3-MT and CTB3-MO or intact CTB3 with nor-toralactone and reactions of the CTB3-MO domain with toralactone. Time-course experiments showed that the appearance of either cercoquinone $\mathrm{C}$ under nonreductive conditions and cercoquinone $\mathrm{D}$ under reductive conditions was coincident with the consumption of toralactone by CTB3-MO and no other intermediates were detectable. Exhaustive attempts to capture the putative intermediate $\mathbf{1 4}$ through methylation by CTB2, an $O$-methyltransferase, were unsuccessful. Reactions contained either CTB2 and CTB3 or CTB2 and CTB3-MO or CTB2 and CTB3-MT, with nor-toralactone or toralactone, respectively, serving as a substrate. As expected, CTB2 was unsuccessful at methylating nortoralactone. Furthermore, it did not alter cercoquinone $\mathrm{C}$ and $\mathrm{D}$ formation by CTB3, as previously observed (Figure S4, Supporting Information).

Structural assignments of cercoquinones $\mathrm{C}$ and $\mathrm{D}$ were supported by HRMS, mass fragmentation, and UV spectral data. Insufficient amounts of material made structural analysis by NMR for these products unfeasible. Structural analogs of both cercoquinones C and $\mathrm{D}$ containing the same quinone cores display similar UV spectra. ${ }^{36}$ The fragmentation data were highly characteristic (Schemes S2 and S3, Supporting Information). As with cercoquinones $\mathrm{A}$ and $\mathrm{B}$, fragmentation ions were observed that comport with the naphthoquinone core. It should be noted that 1,2-naphthoquinones and 1,4-naphthoquinones each fragment in distinct ways. ${ }^{37}$ Additionally, substituents at the $\mathrm{C} 3$ position-the 2 oxopropyl moieties in cercoquinones $\mathrm{C}$ and $\mathrm{D}$-can influence the fragmentation of the quinone ring. ${ }^{37-39}$ Mass fragmentation results for cercoquinones $\mathrm{C}$ and $\mathrm{D}$ are presented in detail in the Supporting Information.

\section{DISCUSSION}

The results presented herein resolve the uncertainties about cercosporin biosynthesis and point to a common biosynthetic pathway for the perylenequinone natural products. ${ }^{25,26} \mathrm{We}$ propose a revised biosynthetic scheme for cercosporin based upon the accumulated metabolites of functional biosynthetic gene knockout strains (Figure 5). First, CTB1 acts according to its established in vitro chemistry producing nor-toralactone $(\mathbf{6}) .{ }^{25}$ The bifunctional enzyme CTB3 methylates nor-toralactone to toralactone (7) before conducting an unusual oxidative aromatic ring opening producing metabolite 14. The $O$ methyltransferase CTB2 is proposed to methylate the nascent OH-6 of intermediate 14blocking further oxidation at this site and yielding compound 15-before the reductase CTB6 reduces the 2-oxopropyl ketone at position C7, giving naphthalene 16. CTB5 is thought to be responsible for homodimerization of intermediate $\mathbf{1 6}$ with CTB7 installing the dioxepine moiety, finally producing cercosporin (1).

We suggest that several of the accumulated metabolites correspond to oxidation products of true on-pathway intermediates. The metabolites cercoquinone A (9) and cercoquinone B (10) from the $\triangle \mathrm{CTB} 6$ and $\triangle \mathrm{CTB} 5$ mutants, respectively, fall into this category. Both quinone products derive from the oxidation of electron-rich naphthalene derivatives. The oxidation of electron-rich aromatic metabolites is common and spontaneous—often leading to stabilized products. ${ }^{40}{ }^{1} \mathrm{H}$ NMR and mass fragmentation data support these structural assignments, especially with respect to the sites of oxidation and methylation. Indeed, the observed 
fragmentation ions agreed with established fragmentation mechanisms for naphthoquinone products. ${ }^{37-39}$ The inability of either $\Delta$ CTB6 or $\Delta$ CTB5 mutants to complement cercosporin activity as secretor-converter pairs bolsters the argument that cercoquinones $\mathrm{A}$ and $\mathrm{B}$ are rapidly formed off-pathway byproducts by spontaneous oxidation.

Interestingly, cercoquinone A displays the loss of a methyl group installed earlier through the activity of the $O$-methyltransferase domain of CTB3. The loss of the methyl at this position is, however, unambiguous given the metabolite's characteristic mass fragmentation pattern; in particular, the retention of the methoxyl in both the ring contraction carbocation and the ring-opened oxonium ion confirms its structural assignment. We envision two possible scenarios that could account for the elimination of this moiety (Scheme 1). In the first possibility, it is simply eliminated as methanol following oxidation and addition of water to the $p$-quinone activated by internal hydrogen bonding. In the second possibility, the methyl position is removed enzymatically. The methoxy at this position is eventually incorporated into the dioxepine functionality of the final product we believe through the activity of CTB7. While the mechanism of this transformation is unknown, by analogy to studies of methylenedioxy formations, ${ }^{41,42}$ it would likely involve oxidative loss. Given the structural similarity of cercoquinone A to the monomeric unit of cercosporin, we suggest that CTB7 could accept this metabolite as an alternative substrate and catalyze the elimination of the methyl in question.

The absence of any extractable cercosporin pathway metabolites in the $\triangle \mathrm{CTB} 2$ and $\Delta \mathrm{CTB} 7$ mutants is notable given that they are absolutely necessary to sustain cercosporin biosynthesis. Naphthoquinone secondary metabolites in fungi have been shown to inhibit fungal growth. ${ }^{43}$ Both $\triangle \mathrm{CTB} 2$ and $\triangle \mathrm{CTB} 7$ mutant strains have more robust growth than either wild-type or mutant strains that accumulate significant amounts of naphthoquinone byproducts. Despite their limited metabolic profiles, both $\triangle \mathrm{CTB} 2$ and $\triangle \mathrm{CTB} 7$ mutant strains appear pigmented while the $\triangle \mathrm{CTB} 1$ mutant strain is colorless. The putative cercosporin pathway intermediates produced in these mutant strains would likely be electron-rich, hydroxylated naphthalene analogs similar to the components of melanin. ${ }^{44}$ Melanin-a crucial biological pigment-is produced in fungi by the polymerization of polyketidederived 1,8-dihydroxynaphthalene monomers. It is a critical metabolite in plant pathogens as it provides mechanical strength to cellular structures responsible for host invasion. ${ }^{45}$ Furthermore, melanin is highly insoluble and would not contribute to the extracted metabolite profiles analyzed in the current study. Together, the data suggest that the metabolites of the $\triangle \mathrm{CTB} 2$ and $\triangle \mathrm{CTB} 7$ mutants are likely captured by a melanin-like selfpolymerization pathway resulting in incorporation into insoluble, pigmented polymers.

As has been shown previously, the in vitro product of CTB1, an NR-PKS, is naphthopyrone nor-toralactone (6). ${ }^{25}$ The evolution of nor-toralactone by the NR-PKS presents an immediate problem. In order to synthesize cercosporin from nor-toralactone, the pyrone ring must be opened; however, there is no apparent enzyme in the CTB cluster that could carry out this transformation through conventional lactonase-like hydrolysis-the common pathway for this type of reaction. ${ }^{46}$ The accumulation of nor-toralactone in the $\Delta$ CTB3c mutant simultaneously confirms the importance of this metabolite while implicating a potential candidate in CTB3 for pyrone ring opening. Furthermore, the successful 
complementation of cercosporin biosynthesis in the $\Delta \mathrm{CTB} 1 / \Delta \mathrm{CTB} 3 \mathrm{c}$ mutant pair corroborates the view that nor-toralactone is an on-pathway intermediate.

CTB3 is an unusual didomain enzyme with an $O$-methyltransferase domain and a flavindependent monooxygenase domain. The in vitro enzymatic reactions presented herein confirm that both domains work in tandem to transform the polyketide product nortoralactone to the on-pathway naphthalene analog 14. Although naphthalene $\mathbf{1 4}$ is not directly observed, it is the implied shared precursor to both cercoquinone $\mathrm{C}(\mathbf{1 2})$ and cercoquinone D (11) whose structures are secured by characteristic mass fragments for both ortho- and para-quinones that confirm the positions of oxidation (Schemes S2 and S3, Supporting Information).

The direct methylation of nor-toralactone to toralactone by CTB3-MT, as well as the appearance of toralactone in reactions of CTB3 with nor-toralactone are strong indicators that toralactone is a true intermediate of the CTB3 catalytic cycle. Furthermore, its appearance in the metabolite profile of the $\triangle \mathrm{CTB} 3 \mathrm{c}$ mutant suggests its biosynthetic importance. The $\triangle \mathrm{CTB} 3 \mathrm{c}$ mutant strain was produced by inserting the knockout cassette into the $5^{\prime}$-terminus of the CTB3 gene corresponding to the CTB3-MO domain. The sequence of the CTB3-MT domain is largely unaltered suggesting the possibility of a functional $O$ methyltransferase. We suspect that the $\triangle \mathrm{CTB} 3 \mathrm{c}$ mutant strain could retain some endogenous CTB3-MT activity resulting in the partial methylation of nor-toralactone in the disruption mutant strain. Alternatively, another $O$-methyltransferase could conceivably convert nortoralactone to toralactone in vivo; however, this hypothetical $O$-methyltransferase could not be CTB2 - the only other methyltransferase in the CTB cluster as it is incapable of this transformation in vitro.

The CTB3-MO domain shares strong primary sequence homology to the canonical $p$ hydroxybenzoate (17) hydrolase family of flavin-dependent monooxygenases. ${ }^{47}$ This is a well-studied family of enzymes with a shared catalytic mechanism and preference for electron-rich aromatic substrates. They are responsible for the hydroxylation of simple phenolic systems with a preference for the ortho and para positions. The reaction cycle proceeds in two halves: reductive and oxidative (Scheme 2a). ${ }^{48}$ The reductive half cycle is initiated with the formation of a ternary complex of enzyme containing oxidized FAD $\left(\mathrm{FAD}_{\mathrm{ox}}, \mathbf{1 8}\right), \mathrm{NAD}(\mathrm{P}) \mathrm{H}$, and substrate. Upon formation of the ternary complex, rapid reduction of $F A D$ by $N A D(P) H$ occurs $\left(F A D_{\text {red }}, 19\right)$. Reduction is contingent on substrate binding or in some cases analogs of the native substrate. $\mathrm{NAD}(\mathrm{P})^{+}$dissociation occurs at a rate similar to that of reduction. The resulting reduced enzyme and substrate complex has significant kinetic stability. In the oxidative half cycle, oxygen reacts with $\mathrm{FAD}_{\text {red }}$, forming the characteristic flavin-C4a-hydroperoxide reactive intermediate (21). The deprotonated flavin-C4a-peroxy species (20) has never been observed in these enzymes. Hydroxylation of the substrate occurs through electrophilic aromatic substitution resulting in an enzymebound product and flavin-C4a-hydroxide (22) complex. Water is eliminated from the flavin species resulting in $\mathrm{FAD}_{\mathrm{ox}}$ and the product is finally released.

We argue that a directly analogous mechanism is at play in the CTB3-MO domain with the enzyme installing hydroxyl at the bridgehead position of toralactone (7, Scheme $2 b)$. The 
hydroxylated intermediate $\mathbf{2 4}$ would be far more susceptible to lactone hydrolysis due to the loss of conjugation and aromaticity and the favorable enolate leaving group. Hydrolysisenzyme catalyzed or spontaneous - would result in acid 25. Loss of carbon dioxide from acid $\mathbf{2 5}$ to generate the $o$-hydroquinone $\mathbf{1 4}$ would likely be spontaneous and rapid with the re-establishment of aromaticity providing a large thermodynamic driving force.

Alternatively, one could also consider a Baeyer-Villiger-like oxidation, with a flavin-C4aperoxy nucleophile attacking the pyrone carbonyl. Ring expansion followed by hydrolysis and loss of carbon dioxide would generate the same product $\mathbf{1 4}$. We consider this mechanism to be unlikely. The $p$-hydroxybenzoate monooxygenase enzyme family invariably proceeds through an electrophilic flavin-C4a-hydroperoxy species while flavoenzyme catalyzed Baeyer-Villiger oxidations rely on the nucleophilic flavin-C4aperoxy species. ${ }^{49}$

There is precedence for $p$-hydroxybenzoate monooxygenase family members participating in oxidative aromatic ring cleavage. The enzymes 2-methyl-3-hydroxypyridine-5-carboxylic acid (MHPC, 26a) oxygenase (MHPCO) and 5-pyridoxic acid (5PA, 26b) oxygenase (5PAO) are flavoenzymes of the $p$-hydroxybenzoate monooxygenase family catalyzing aromatic hydroxylation and subsequent aromatic ring cleavage reactions (Scheme 2c). ${ }^{50}$ Like conventional flavin monooxygenases, these enzymes use the flavin-C4a-hydroperoxy reactive intermediate (21) in their initial electrophilic aromatic oxidation; however, they undergo a subsequent ring-opening reaction. The mechanism of ring opening has not been definitively established, but it is known that ring opening is enzyme catalyzed in MHPCO and involves the addition of water to the carbonyl intermediate (27a). ${ }^{51}$

The evolution of cercoquinones $\mathrm{C}$ and $\mathrm{D}$ and their putative shared intermediate $\mathbf{1 4}$ are in keeping with the proposed mechanism (Scheme $2 b$ ). The transfer of a hydride equivalent from dihydroquinone $\mathbf{1 4}$ to $\mathrm{FAD}_{\mathrm{ox}}$, while not anticipated, is not unreasonable. In the absence of a reductive environment, one could envision this oxidation is the dominant pathway towards cercoquinone $\mathrm{C}$ as we observe. Furthermore, the observed consumption of nortoralactone by CTB3-MO implies an ortho-quinone intermediate. Ortho-quinone analogs of cercoquinone $\mathrm{C}$ have been used previously to access the perylenequinone core through simple coupling under acidic conditions. ${ }^{52-54}$ Assuming nor-toralactone serves as an alternative substrate for CTB3-MO, the observed but uncharacterized dimeric products could likely arise through analogous couplings.

Under reductive conditions (DTT), cercoquinone $\mathrm{C}$ upon release would be rapidly reduced back to species 14. Electron-rich naphthalene species such as compound $\mathbf{1 4}$ are highly prone to spontaneous oxidation. One could envision the oxidation of compound 14 to cercoquinone $\mathrm{D}$ as being rapid and complete, even under reductive conditions. The apparent instability of intermediate $\mathbf{1 4}$ could explain the observed preference for oxidation of orthoquinone cercosporin $\mathrm{C}$. In the quinone oxidation state, this species is protected from subsequent oxidation, ensuring biosynthetic fidelity.

Structural analysis of known fungal perylenequinone natural products reveals several common features among this family of metabolites (Figure 6) ${ }^{54}$ First, the $7,7^{\prime}$ moieties are derived from 2-oxypropyl side chains. Second, the 2, $2^{\prime}$ substituents are always methoxyl, 
or in the case of cercosporin a derivative thereof. Third, the $6,6^{\prime}$ substituents are always methoxyl or derivatives thereof. Fourth, the perylenequinone core architecture remains unaltered. These core structural features suggest a common biosynthetic pathway for the perylenequinone metabolites. Indeed, the activities of CTB1, CTB3, CTB2, and CTB5 delineated here would account for each of these features with CTB1 producing the common intermediate nor-toralactone, CTB3 methylating this intermediate at the $\mathrm{OH}-2$ and opening the pyrone ring thereby installing a $\mathrm{C6}-\mathrm{OH}, \mathrm{CTB} 2$ methylating the nascent $\mathrm{C6}-\mathrm{OH}$, and CTB5 catalyzing dimerization yielding the perylenequinone carbon core. Interestingly, homologs of each of these enzymes are found in known gene clusters most similar to the CTB cluster. Of these clusters, only one is linked to a known product, hypocrellin A (13). ${ }^{35}$ Hypocrellin A differs from cercosporin in three key features: the $7,7^{\prime}$ substituents are retained in the 2-oxypropyl oxidation state and are linked through an intramolecular aldol reaction, the $2,2^{\prime}$ substituents are methoxyl, and the compound adopts the opposite atropisomeric configuration. Comparing the CTB and hypocrellin A biosynthetic clusters, CTB6 and CTB7 appear unique to the CTB cluster. Given that these enzymes presumptively reduce the 2-oxypropyl substituents and install the dioxepine moiety, respectively, it makes sense that they would be missing in the hypocrellin A cluster, where these structural features are not present. Altogether, these data imply that perylenequinone natural products proceed through a common biosynthetic pathway in which nor-toralactone is initially produced and further processed by a CTB3 didomain homolog. Given its unique coupled activities, CTB3 and its homologs make appealing targets for antifungal agents.

\section{CONCLUSION}

Here we resolve the long-standing ambiguity of cercosporin biosynthesis and propose an alternative biosynthetic pathway. We demonstrate that the naphthoquinone nor-toralactone is an essential intermediate of the cercosporin pathway, further corroborating the previously observed in vitro activity of the NR-PKS CTB $1 .{ }^{25}$ We also characterize the activity of the unusual didomain protein CTB3 showing that its flavin-dependent monooxygenase domain is responsible for an uncommon oxidative aromatic cleavage. Together, these findings further not only our understanding of perylenequinone biosynthesis but also expand the known biochemical repertoire of flavin-dependent monooxygenases-an important class of enzymes in both primary and secondary metabolism. While addressing in detail the initial steps of cercosporin biosynthesis, data presented only partially resolve the later portion of the biosynthetic pathway. The crucial perylenequinone dimerization step(s) and how the absolute configurations of the atropisomers are set remain intriguing, unanswered mechanistic questions. Given the importance of cercosporin and its congeners for plant pathogenicity, disruption of these steps or the rare confluence of CTB3 activities present opportunities for the development of selective antifungal agents against Cercospora species.

\section{Supplementary Material}

Refer to Web version on PubMed Central for supplementary material. 


\section{Acknowledgments}

We thank Dr. Kuang-Ren Chung (National Chung Hsing University, Taichung, Taiwan) for providing C. nicotianae (ATCC $^{(} 18366^{\mathrm{TM}}$ ) wild type and CTB gene cluster knockout strains. We thank Philip A. Storm and Callie R. HuittRoehl (Department of Chemistry, Johns Hopkins University, Baltimore, MD, USA) for helpful conversations. The authors declare no financial conflicts of interest. This work was supported by NIH Grant ES001670.

\section{References}

1. Daub ME. Phytopathology. 1981; 71:213-213.

2. Morgan BJ, Dey S, Johnson SW, Kozlowski MC. J Am Chem Soc. 2009; 131:9413-9425. [PubMed: 19489582]

3. Morgan BJ, Mulrooney CA, Kozlowski MC. J Org Chem. 2010; 75:44-56. [PubMed: 19894744]

4. Daub ME, Herrero S, Chung KR. FEMS Microbiol Lett. 2005; 252:197-206. [PubMed: 16165316]

5. Kuyama S, Tamura T. J Am Chem Soc. 1957; 79:5725-5726.

6. Kuyama S, Tamura T. J Am Chem Soc. 1957; 79:5726-5729.

7. Lousberg RJJC, Weiss U, Salemink CA, Arnone A, Merlini L, Nasini G. J Chem Soc D. 1971:14631464.

8. Yamazaki S, Ogawa T. Agric Biol Chem. 1972; 36:1707-1718.

9. Nasini G, Merlini L, Andreetti GD, Bocelli G, Sgarabotto P. Tetrahedron. 1982; 38:2787-2796.

10. Daub ME, Hangarter RP. Plant Physiol. 1983; 73:855-857. [PubMed: 16663313]

11. Dobrowolski DC, Foote CS. Angew Chem Int Ed Engl. 1983; 22:720-721.

12. Daub ME. Plant Physiol. 1982; 69:1361-1364. [PubMed: 16662404]

13. Daub ME, Briggs SP. Plant Physiol. 1983; 71:763-766. [PubMed: 16662903]

14. Daub ME, Ehrenshaft M. Annu Rev Phytopathol. 2000; 38:461-490. [PubMed: 11701851]

15. Yamazaki S, Okubo A, Akiyama Y, Fuwa K. Agric Biol Chem. 1975; 39:287-288.

16. Fajola AO. Physiol Plant Pathol. 1978; 13:157-164.

17. Daub ME. Phytopathology. 1982; 72:370-374.

18. Choquer M, Dekkers KL, Chen HQ, Cao LH, Ueng PP, Daub ME, Chung KR. Mol Plant Microbe Interact. 2005; 18:468-476. [PubMed: 15915645]

19. Choquer M, Lee MH, Bau HJ, Chung KR. FEBS Lett. 2007; 581:489-494. [PubMed: 17250832]

20. Dekkers KL, You BJ, Gowda VS, Liao HL, Lee MH, Bau HH, Ueng PP, Chung KR. Fungal Genet Biol. 2007; 44:444-454. [PubMed: 17074519]

21. Daub ME, Leisman GB, Clark RA, Bowden EF. Proc Natl Acad Sci USA. 1992; 89:9588-9592. [PubMed: 1409670]

22. Leisman GB, Daub ME. Photochem Photobiol. 1992; 55:373-379.

23. Sollod CC, Jenns AE, Daub ME. Appl Environ Microbiol. 1992; 58:444-449. [PubMed: 1610167]

24. Okubo A, Yamazaki S, Fuwa K. Agric Biol Chem. 1975; 39:1173-1175.

25. Newman AG, Vagstad AL, Belecki K, Scheerer JR, Townsend CA. Chem Commun. 2012; 48:11772-11774.

26. Chen HQ, Lee MH, Daub ME, Chung KR. Mol Microbiol. 2007; 64:755-770. [PubMed: 17462021]

27. Chen HQ, Lee MH, Chung KR. Microbiology. 2007; 153:2781-2790. [PubMed: 17660442]

28. Chung KR, Jenns AE, Ehrenshaft M, Daub ME. Mol Gen Genet. 1999; 262:382-389. [PubMed: 10517336]

29. Chung KR, Daub ME, Kuchler K, Schuller C. Biochem Biophys Res Commun. 2003; 302:302310. [PubMed: 12604346]

30. Herrero S, Amnuaykanjanasin A, Daub ME. FEMS Microbiol Lett. 2007; 275:326-337. [PubMed: 17850326]

31. Akache B, Wu K, Turcotte B. Nucleic Acids Res. 2001; 29:2181-2190. [PubMed: 11353088]

32. Ehrenshaft M, Upchurch RG. Appl Environ Microbiol. 1991; 57:2671-2676. [PubMed: 16348553]

J Am Chem Soc. Author manuscript; available in PMC 2016 November 30. 
33. Jenns AE, Daub ME. Phytopathology. 1995; 85:906-912.

34. Weber T, Blin K, Duddela S, Krug D, Kim HU, Bruccoleri R, Lee SY, Fischbach MA, Muller R, Wohlleben W, Breitling R, Takano E, Medema MH. Nucleic Acids Res. 2015; 43:W237-243. [PubMed: 25948579]

35. Yang H, Wang Y, Zhang Z, Yan R, Zhu D. Genome Announc. 2014; 2:e00011-14. [PubMed: 24503982]

36. Ebnother A, Meijer TM, Schmid H. Helv Chim Acta. 1952; 35:910-928.

37. Vessecchi R, Emery FS, Lopes NP, Galembeck SE. Rapid Commun Mass Spectrom. 2013; 27:816824. [PubMed: 23495028]

38. Vessecchi R, Emery FS, Galembeck SE, Lopes NP. Rapid Commun Mass Spectrom. 2010; 24:2101-2108. [PubMed: 20552691]

39. Vessecchi R, Emery FS, Galembeck SE, Lopes NP. J Mass Spectrom. 2012; 47:1648-1659. [PubMed: 23280754]

40. Wheeler MH, Klich MA. Pestic Biochem Physiol. 1995; 52:125-136.

41. Bauer W, Zenk MH. Tetrahedron Lett. 1989; 30:5257-5260.

42. Rueffer M, Zenk MH. Phytochemistry. 1994; 36:1219-1223.

43. Medentsev AG, Akimenko VK. Phytochemistry. 1998; 47:935-959. [PubMed: 9564730]

44. Eisenman HC, Casadevall A. Appl Microbiol Biotechnol. 2012; 93:931-940. [PubMed: 22173481]

45. Chen Z, Nunes MA, Silva MC, Rodrigues CJ Jr. Mycologia. 2004; 96:1199-1208. [PubMed: 21148942]

46. Thomas PW, Stone EM, Costello AL, Tierney DL, Fast W. Biochemistry. 2005; 44:7559-7569. [PubMed: 15895999]

47. Huijbers MME, Montersino S, Westphal AH, Tischler D, van Berkel WJH. Arch Biochem Biophys. 2014; 544:2-17. [PubMed: 24361254]

48. Entsch B, van Berkel WJ. The FASEB Journal. 1995; 9:476-483. [PubMed: 7737455]

49. Mirza IA, Yachnin BJ, Wang S, Grosse S, Bergeron H, Imura A, Iwaki H, Hasegawa Y, Lau PC, Berghuis AM. J Am Chem Soc. 2009; 131:8848-8854. [PubMed: 19385644]

50. Chaiyen P. Arch Biochem Biophys. 2010; 493:62-70. [PubMed: 19728986]

51. Chaiyen P, Brissette P, Ballou DP, Massey V. Biochemistry. 1997; 36:8060-8070. [PubMed: 9201954]

52. Hauser FM, Sengupta D, Corlett SA. J Org Chem. 1994; 59:1967-1969.

53. Merlic CA, Aldrich CC, Albaneze-Walker J, Saghatelian A, Mammen J. J Org Chem. 2001; 66:1297-1309. [PubMed: 11312960]

54. Mulrooney CA, Morgan BJ, Li X, Kozlowski MC. J Org Chem. 2010; 75:16-29. [PubMed: 19894746] 
a

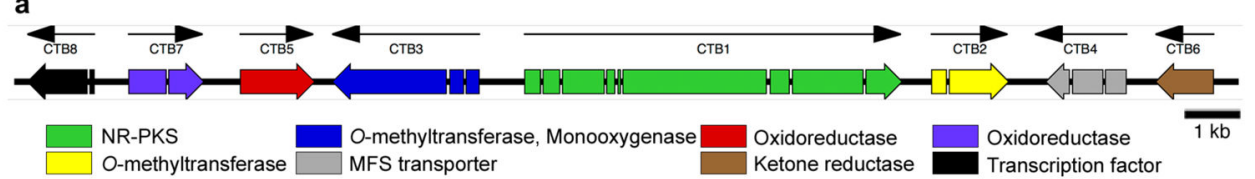

b acetyl-CoA + 6 malonyl-CoA

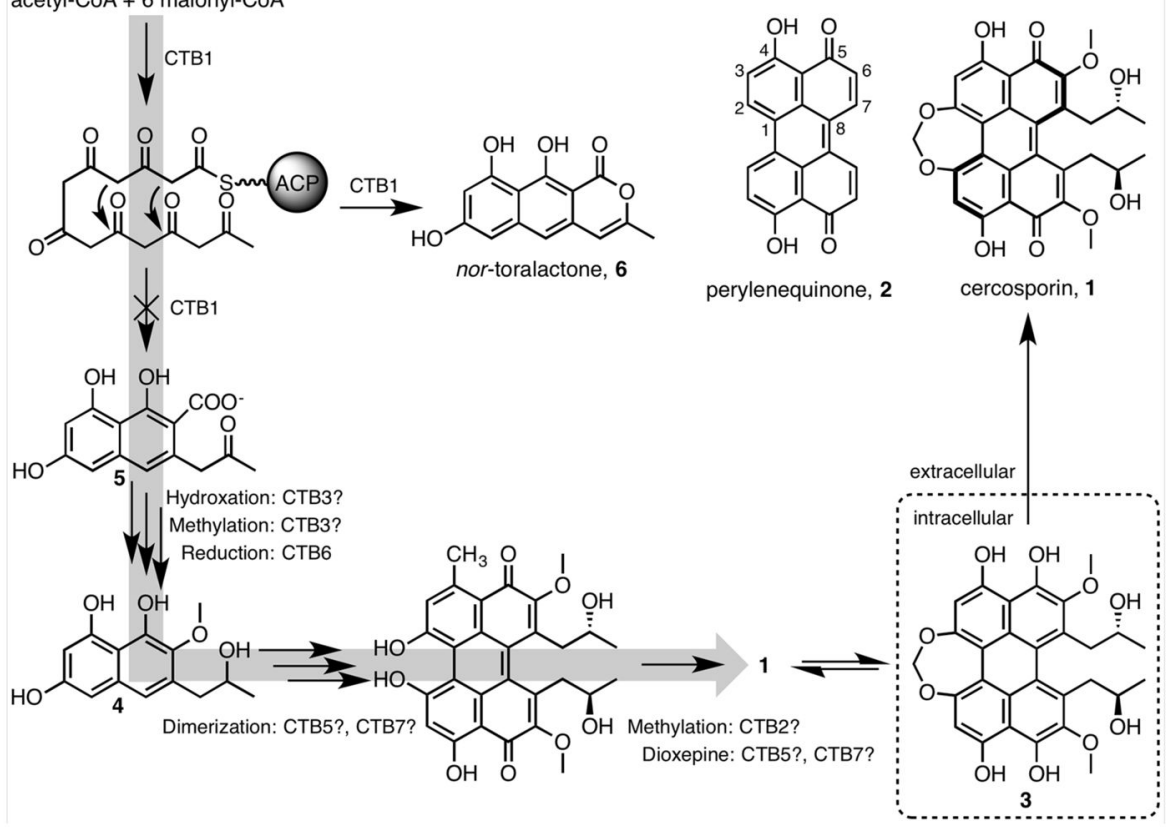

Figure 1.

The currently proposed cercosporin biosynthetic pathway. (a) The cercosporin toxin biosynthetic (CTB) gene cluster has been identified in $C$. nicotianae. (b) The proposed cercosporin biosynthesis hinges upon the formation of carboxylic acid $\mathbf{5}$ by the NR-PKS CTB1. The direct product of CTB1 is nor-toralactone (6), precluding the proposed biosynthetic scheme. 


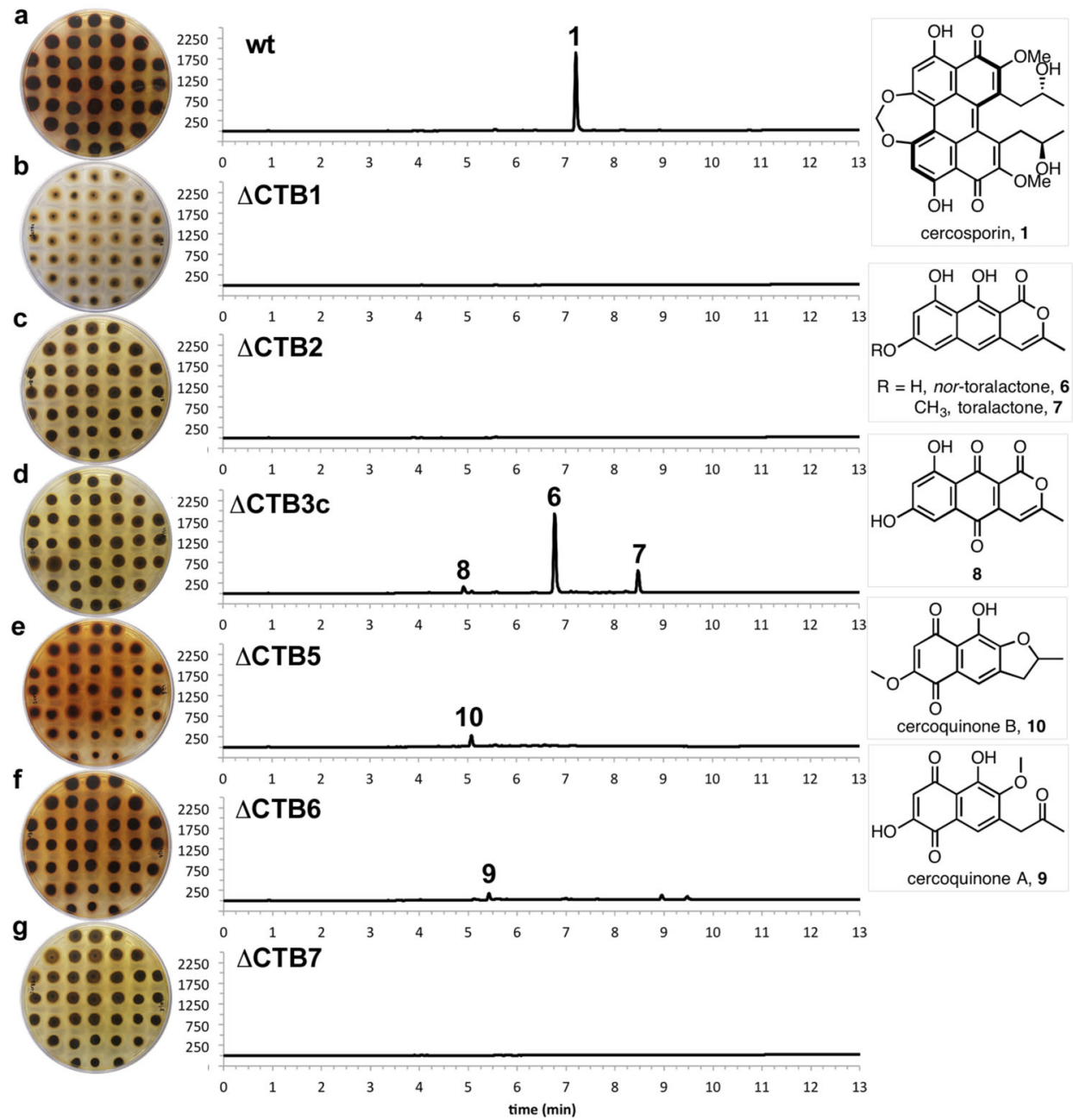

Figure 2.

Metabolic profiles of CTB gene cluster mutant strains. Chromatograms at $250 \mathrm{~nm}$ of extracted metabolite profiles for (a) wild-type, (b) $\Delta$ CTB1, (c) $\triangle \mathrm{CTB} 2$, (d) $\triangle \mathrm{CTB} 3 \mathrm{c}$, (e) $\triangle \mathrm{CTB} 5$, (f) $\triangle \mathrm{CTB} 6$, and (g) $\triangle \mathrm{CTB} 7$ strains displayed along with images of the mycelia for each strain. The metabolites were prepared at a concentration of $10 \mathrm{~cm}^{2}$ colony surface area per $\mathrm{mL}$ in methanol. Identified cercosporin intermediate metabolites are displayed. 

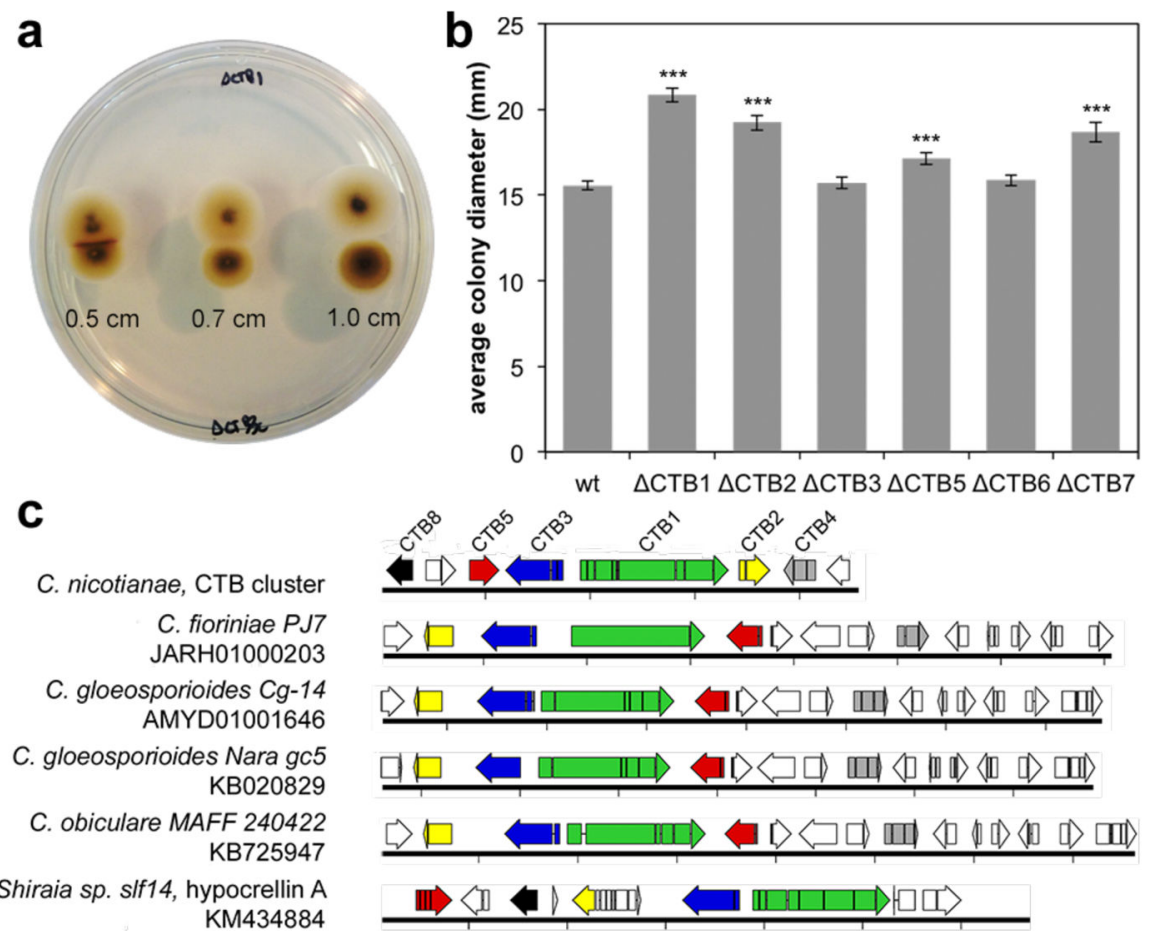

Figure 3.

Phenotypic and genetic analysis of the CTB cluster. (a) Cercosporin biosynthesis was complemented at the colony-colony interface of the $\Delta \mathrm{CTB} 1 / \Delta \mathrm{CTB} 3 \mathrm{c}$ mutant pair (top/ bottom, respectively). The numbers indicate the distance that colonies were inoculated apart from one another in $\mathrm{cm}$. (b) Average colony diameters of CTB disruption mutants are displayed $(n=37)$. Error bars represent the $95 \%$ confidence interval. Stars indicate statistically significant difference from wild-type ( $<<0.01)$. (c) Comparison of gene clusters similar to the CTB gene cluster of $C$. nicotianae (top). 


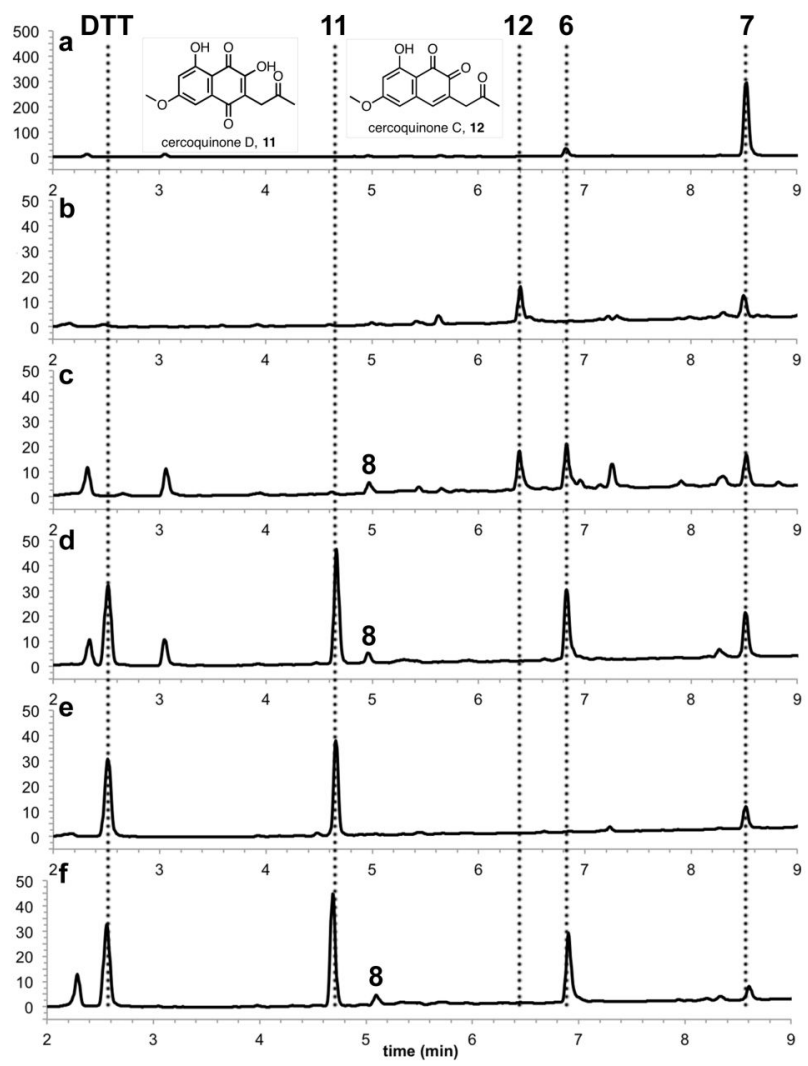

Figure 4.

Product profiles of in vitro reactions of CTB3. The $280 \mathrm{~nm}$ chromatograms of the following reactions are displayed: (a) CTB3-MT with nor-toralactone, (b) CTB3-MO with toralactone, (c) CTB3-MT and CTB3-MO with nor-toralactone, (d) CTB3-MT and CTB3-MO with nortoralactone under reductive conditions, (e) CTB3-MO with toralactone under reductive conditions, and (f) CTB3 with nor-toralactone under reductive conditions. Peaks for nortoralactone (6) and toralactone (7) are indicated along with peaks for products cercoquinone C (12) and cercoquinone D (11). A peak for DTT and other cosubstrates are observed, as applicable. 


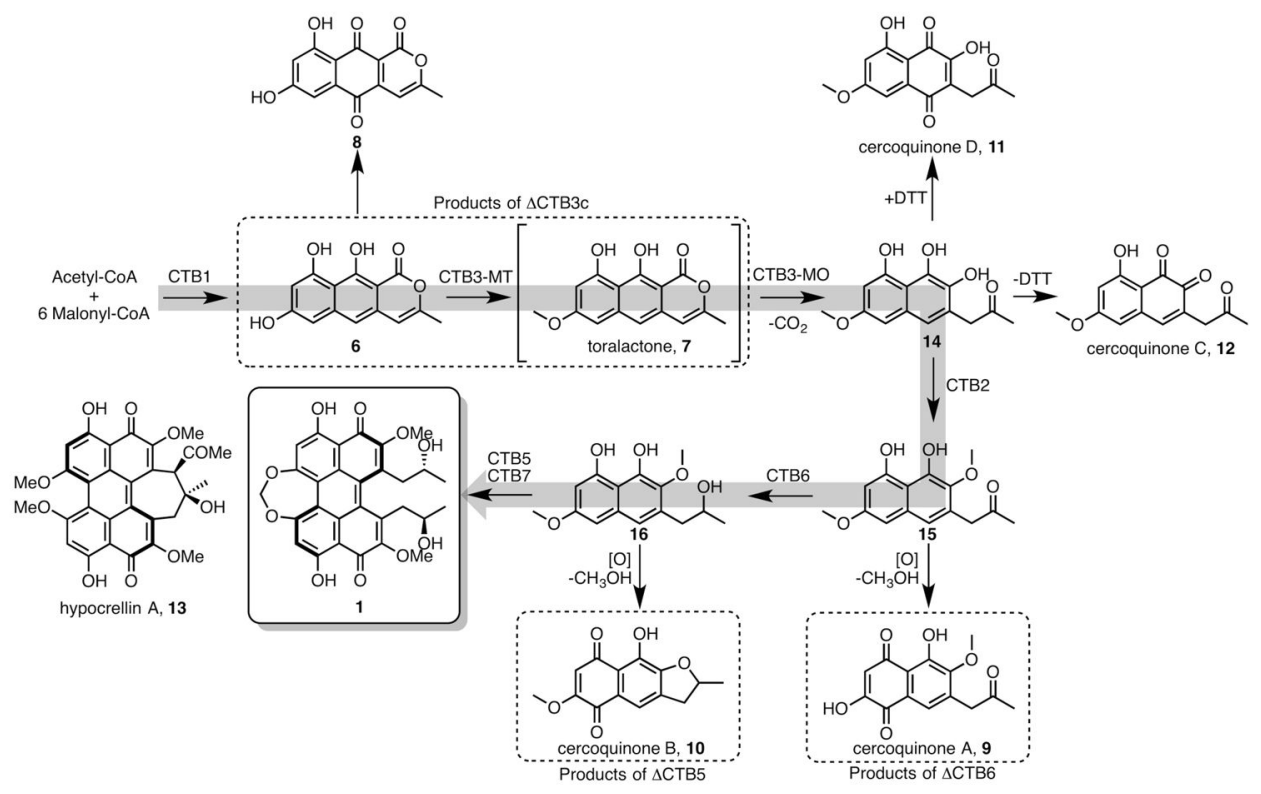

Figure 5.

Proposed cercosporin biosynthetic pathway. On the strength of observed pathway intermediates, in vitro chemistry, phenotypic, genetic, and pairwise complementation a revised biosynthetic scheme for cercosporin is presented. 

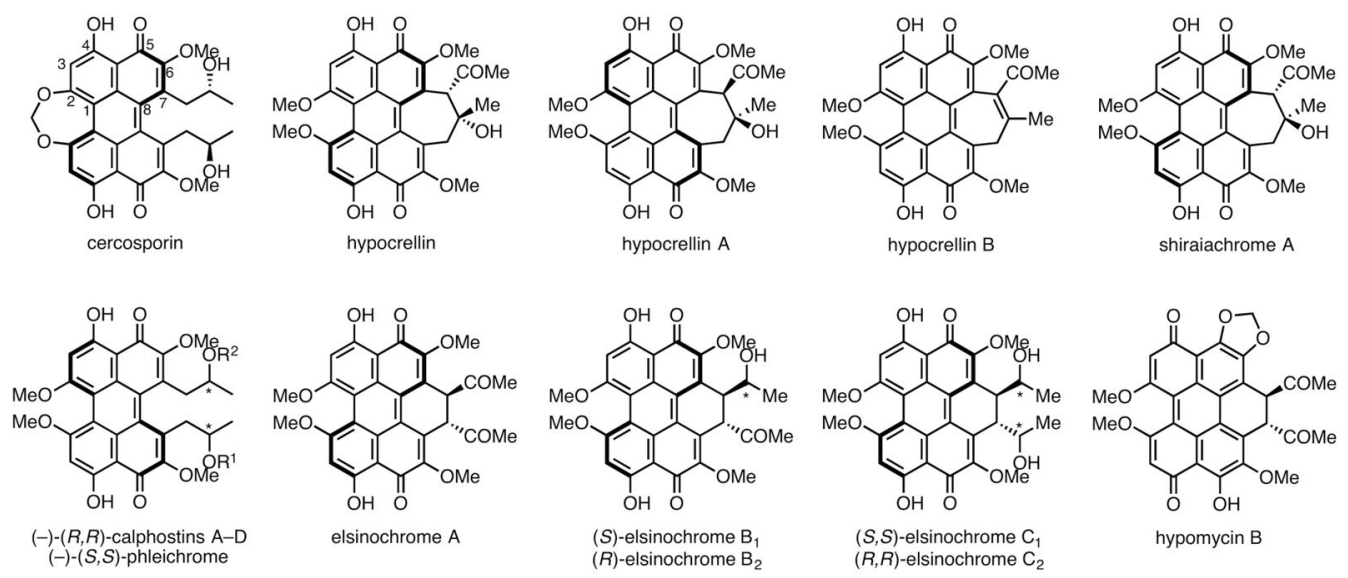

Figure 6.

Structures of known fungal perylenequinone natural products. Common architectural features are observed in the family. The occurrence of methyoxy at $2,2^{\prime}$ and $6,6^{\prime}$ positions are invariable. As is the 2-oxypropyl derivatives at $7,7^{\prime}$. The formation of the perylenequinone core also appears to be conserved. 

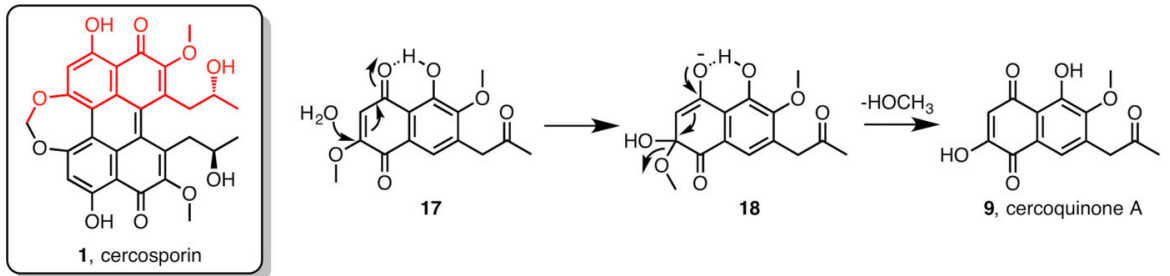

Scheme 1.

Proposed formation of cercoquinone A. Two mechanisms were considered. Addition of water followed by elimination of methanol (pictured) or enzymatic demethylation.

Cercosporin is shown with a monomeric unit shown in red. CTB7 is postulated to form the dioxepine ring, a transformation that would require the elimination of a methyl group at the position of demethylation in cercoquinone A. 


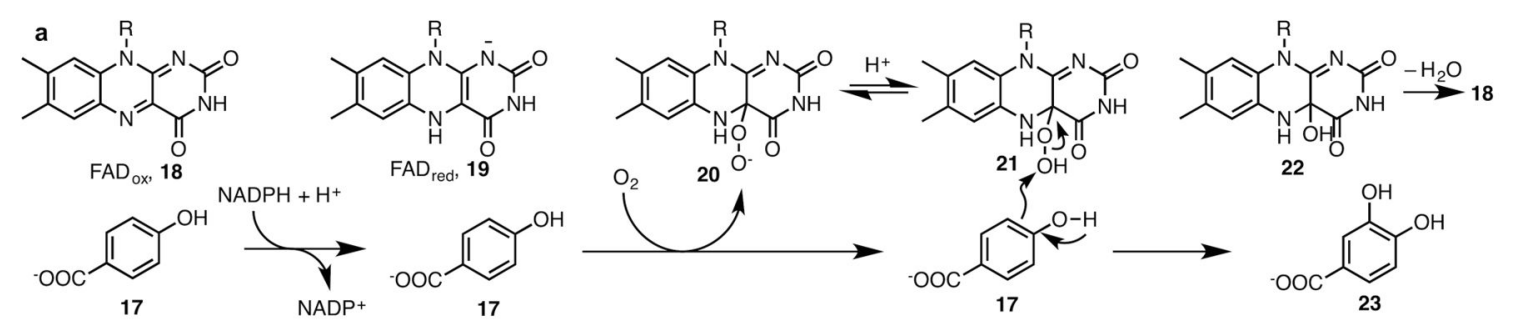

b

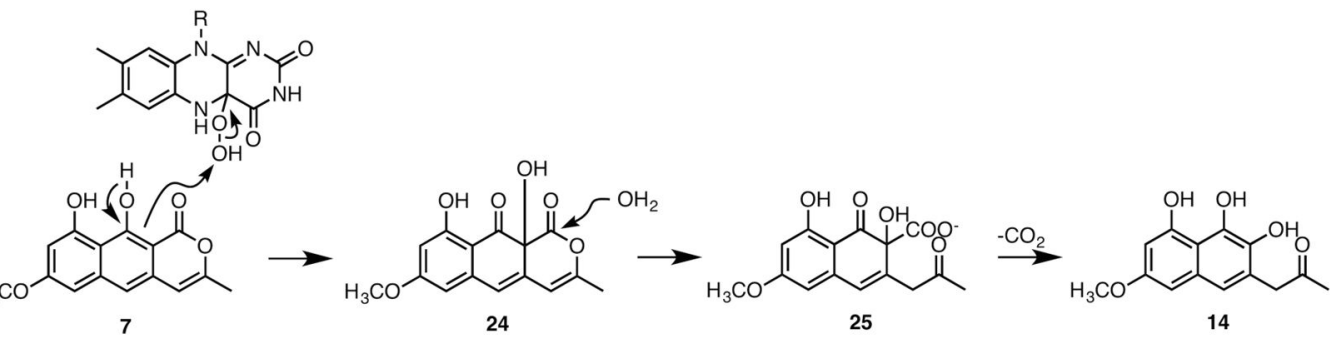

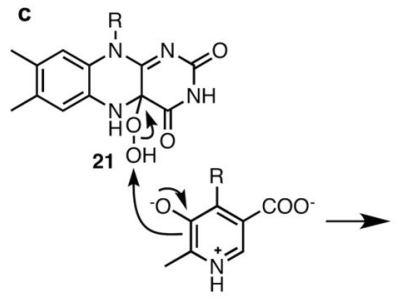

$\mathrm{R}=\mathrm{H}, \mathrm{MHPC}, 26 \mathrm{a}$ $\mathrm{R}=\mathrm{CH}_{2} \mathrm{OH}, 5 \mathrm{PA}, \mathbf{2 6 b}$
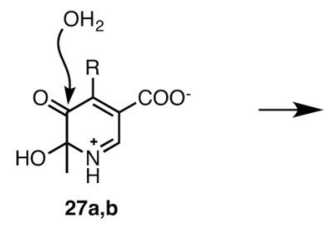
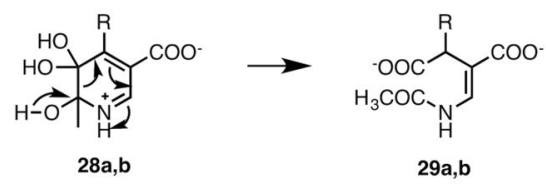

Scheme 2.

Proposed mechanism for CTB3 flavin-dependent monooxygenase domain. (a) General mechanism of $p$-hydroxybenzoate (17) hydroxylase family members. (b) Proposed mechanism for CTB3 catalyzed oxidative aromatic ring cleavage of toralactone (7). (c) Mechanism of MHPCO and 5PAO oxidative aromatic ring cleavage. 

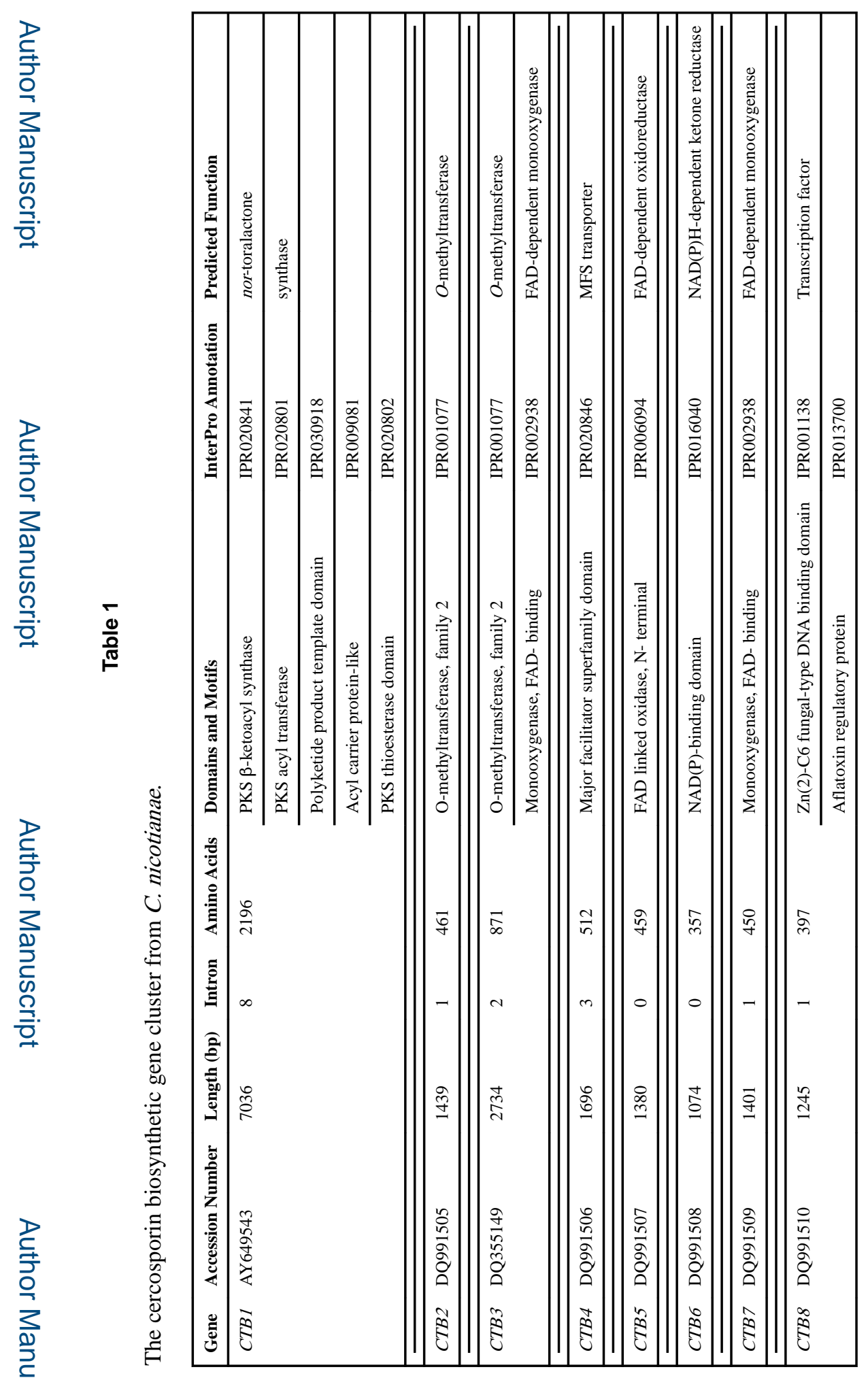

J Am Chem Soc. Author manuscript; available in PMC 2016 November 30. 